\title{
Evaluation of structural changes of HT-PEFC electrodes from in-situ synchrotron $\mathrm{X}$-ray radiographs
}

\author{
Dieter Froning ${ }^{\mathrm{a}, *}$, Wiebke Maier ${ }^{\mathrm{a}}$, Jennifer Groß $^{\mathrm{a}, \mathrm{b}}$, Tobias Arlt ${ }^{\mathrm{c}}$, Ingo Manke ${ }^{\mathrm{c}}$, Werner Lehnert $^{\mathrm{a}, \mathrm{d}}$ \\ ${ }^{a}$ Forschungszentrum Jülich GmbH, Institute of Energy and Climate Research, IEK-3: Electrochemical Process Engineering, Jülich, Germany \\ ${ }^{b}$ Present address: Forschungszentrum Jülich GmbH, Jülich Supercomputing Centre, Jülich, Germany \\ ${ }^{c}$ Helmholtz-Zentrum Berlin GmbH, Institute of Applied Materials, Berlin, Germany \\ ${ }^{d}$ Modeling in Electrochemical Process Engineering, RWTH Aachen University, Germany
}

\begin{abstract}
One of the main goals for improvement of high-temperature polymer electrolyte fuel cells (HT-PEFCs) is the increase of the fuel cell performance under different operating conditions. We investigated the correlation between operating conditions and structural changes in the electrodes by means of in-situ through-plane synchrotron X-ray radiography. From the radiographs it is possible to clearly distinguish between the electrode crack structure under the ribs and under the channels of the flow field. We present a statistical method to analyze these crack structures. For this purpose a 'radar' method was developed in order to obtain the width of the cracks at many different locations and the distribution of crack widths. We found a strong influence of the operating conditions on the crack structure and a different behavior of cracks located beneath the ribs and beneath the channels.
\end{abstract}

Keywords: HT-PEFC, electrode crack structure, crack width, synchrotron X-ray radiography

\section{Introduction}

High temperature polymer electrolyte fuel cells (HTPEFCs) are typically operated at temperatures between 160 and $180{ }^{\circ} \mathrm{C}$. Like classical PEFCs they are based on polymer electrolyte membranes. For the manufacturing of membrane electrode assemblies (MEA) several methods can be applied. Common to all of them is the production of a catalyst paste. The latter is applied either directly onto the membrane or onto the gas diffusion layer (GDL) by means of several coating techniques. The drying process leads to a characteristic structure of cracks in the membrane electrode assembly (MEA) as shown in Figure 1 [1, 2]. The morphology of the crack structure depends on the thickness of the electrode, the temperature and duration of the drying process, and the humidity in the laboratory. Not only the electrochemical active area is reduced by the cracks, in particular they affect the water transport in PEFCs [3, 4, 5]. Hence, an impact of the cracks on cell performance cannot be neglected. Cracks in the manufactured electrodes can be easily characterized before stack

\footnotetext{
*Corresponding author; tel.: +49 246161 6676; fax: +49 24616 6695

Email address: d.froning@fz-juelich.de (Dieter Froning)
}

assembly using several image processing techniques, e.g. scanning electron microscopy (SEM), transmission electron microscopy (TEM), light microscopy (see Figure 1) but this techniques don't contain information regarding changing properties of the cracks and their impact on the cell performance under dynamic operation of the fuel cells.

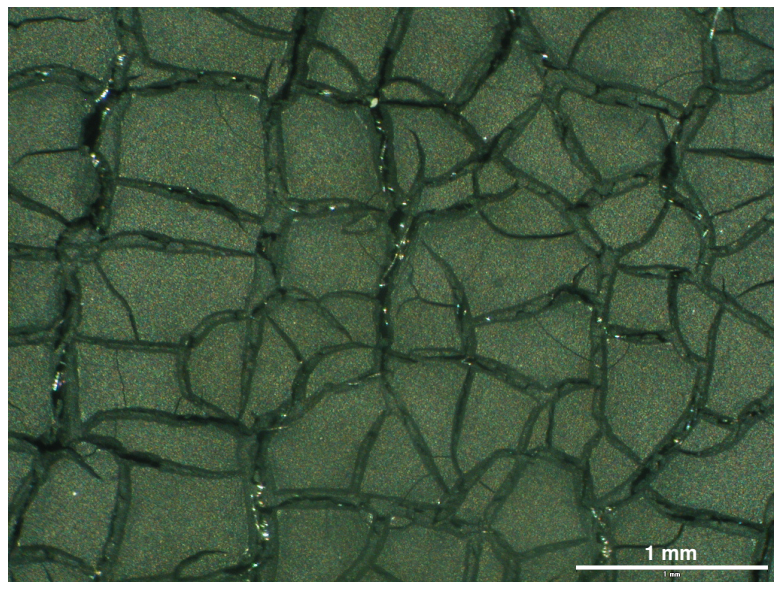

Figure 1: Microscope image of cracks and blocks visible in the catalyst layer of a gas diffusion electrode 
Manke et al. [6], Sasabe [7], Deevanhxay et al. [8], Markötter et al. [5], and Eller et al. [9] applied in-situ $\mathrm{X}$-ray radiography on PEFCs to investigate water distribution in gas diffusion layers and micro-porous layers of PEFCs under varying operating conditions.

The fraction of cracks related to the whole area can easily be obtained by just counting the pixels after converting the image to black/white (BW) [1]. Other investigations are targeting the pore structure of the catalyst in nano scales. However, it is desirable to have an analytical method to quantify the morphology (or structure) of the cracks in the micro structure as it is visible in Figure 1

The importance of crack structures is well-known in various fields. Novák et al. [10] investigated cracked clay soil. There are numerous methods describing the movement of water transport in cracked soil [10]. Basically they can be separated into few classes of methods. Transport models based on crack networks suffer from relatively poor results in the application of water transport in cracked soil [10]. The benefit of models which are using statistical properties of cracks (e.g., depths and widths) is that they do not require detailed knowledge of the crack system. Novák et al. [10] developed a detailed mathematical model to describe the water transport in the particular kind of cracks in swelling soil. Their method belongs to another class of methods featuring detailed physical modeling. Landry and Karpyn [11] investigated in fluid flow in fractured permeable media. For this purpose, the structure of porous polyethylene rods with $0.127 \mathrm{~cm}$ in diameter was obtained via Xray radiography. Landry and Karpyn investigated in the cracks as means of fluid transport.

Regarding the manufacturing of PEFC MEAs, Wannek et al. [1] observed a relationship between the morphology of the cracks and the dispersing technology of applying the catalyst material on the decal substrate. They evaluated the amount of cracks in the dry catalyst layer related to the total area. Hizir et al. [12] used the same method to define the crack density in the micro porous layer (MPL) and the catalyst layer (CL). For characterizing the morphology oft the surface they took a set of profiles obtained with optical profilometry. With this method they got a set of height profiles parallel to the coordinate axes and they evaluated six amplitude parameters defined by the British Standards [13].

Sasabe et al. [7], and Deevanhxay et al. [8] studied cracks in MPLs and GDLs which are another components in PEFCs. They used soft X-ray radiography for their studies. The breakthrough of water from the electrode into the GDL and its flow paths in MPL and GDL were analyzed.
Kundu et al. [4] classified the physical processes leading to degradation of fuel cells in presence of cracks. They identified cracking in the catalyst layer as one - but not the only one - defect in the manufacturing process of MEAs affecting the quality. As a consequence, comprehensive investigations are required not only on experimental studies but also on modeling the physical processes taking place in the wide area of degradation of the catalyst layer.

Pfrang et al. [14] observed cracks in the cathode CL via $\mathrm{X}$-ray radiography. After marking the cracks manually in their images they investigated the 3D characteristic of the cracks regarding their volume in the $\mathrm{CL}$. The crack density was also monitored.

Investigations on the design of CL often address the three-dimensional structure [15] or its effective parameters [16]. Cracks in the CL affect the interface between the CL and the MPL/GDL.

Arlt et al. [17] investigated the Pt and Ru concentration in the catalyst layers of a direct methanol fuel cell (DMFC). They found differences between areas located under the land and areas located under the ribs of the bipolar plate. Because Pt is located on both CL at the anode and cathode side, cracks in both CLs were observed as overlaid structure in the Pt images. On the other hand, only the cracks on the cathode side were found in the Ru images.

In contrast to the three-dimensional methods for crack analysis mentioned above we developed a novel method for quantitative analysis of the two-dimensional structures which can be observed already with light microscope or SEM images.

In this work we present investigations on statistical properties of the catalyst crack structures. We are able to obtain characteristics of the structure of cracks and blocks from black/white (BW) images. For this purpose we created objects representing the blocks which were extracted from the BW images via classical methods of image processing. Every block is stored in a small image that can be analyzed individually. The object oriented analysis allows us to calculate structural information from them.

The area of the blocks can be obtained from the object image as well as the length of the circumference of the blocks. For the case if some fluid might interact with the catalyst material from within the cracks the borderline along the cracks might be of interest. According to the definition of a hydraulic diameter known in fluid dynamics we introduced the hydraulic diameter of a block as a measure of the shape of the block by defining $d_{\text {hyd }}=4 A / U$ - given the area $A$ and the circumference $U$-which is the geometric diameter in case of 
a circle. The orientation of the blocks can be calculated. Blocks which are of irregular shape can be identified.

All the values above are calculated from every block. That ends up in a distribution of the characteristics mentioned above. The blocks overlapping the border of the image are neglected in stochastic evaluation because they would distort the characteristics.

The total length of all cracks can be estimated using the assumption that every crack is adjoined to two blocks at every position along the crack. Hence, the sum of the circumferences of all blocks divided by two gives the total length of the cracks. In this case the outer blocks overlapping the image borders need to be taken into account - but without the image border itself.

Last but not least the width of the cracks can be obtained from the BW images. Other than the measures above the width is not calculated merely from the single objects representing the blocks but from the BW image. Furthermore the number of evaluations is independent from the number of blocks in the image.

The nature of the images from the BESSY synchrotron limits their size and therefore the number of blocks which can be found in a single image - an example is shown in Figure 2. With this restriction one has to be careful in evaluating the block related measures because their number might be too small for statistical analysis. But this restriction does not hold for the analysis of the crack widths as it will be argued later. In the running fuel cell we observed a widening of the cracks under the channels, while the widths of the cracks under the ribs remained about the same. Therefore we focused our investigations on the analysis of the widths of the cracks during cell operation.

From the in-situ images similar cracks were observed as they are known from the drying process of the catalyst layer (Figure 2). Two regions of the fuel cell can be identified in the gray level image: one bright region under the gas channel, and one dark region under the ribs of the bipolar plate. Because of the construction of the stack it has to be considered that the morphological structure might be different in the channel regions and in the land regions. In Figure 2 it can be seen that the remaining regions are too narrow to provide enough blocks which are not cut by some border to allow a statistical evaluation of the blocks. But the crack widths can be evaluated at much more locations so they can be evaluated under stochastic view.

The methods for analyzing the structure are also applicable for classical PEM fuel cells, i.e. PEFCs operating at up to $90{ }^{\circ} \mathrm{C}$ or DMFC. Both types of fuel cells are based on similar membranes.

In this work, we describe the methods of our struc-

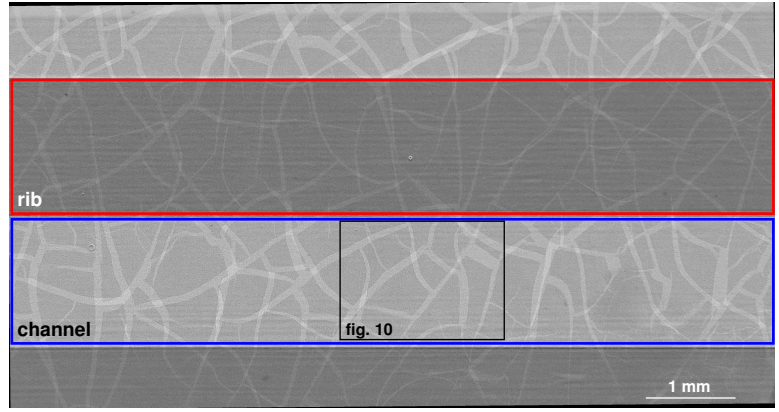

Figure 2: Radiography of a $8.5 \mathrm{~mm} \times 4.5 \mathrm{~mm}$ section of an in-situ HT-PEFC at $600 \mathrm{~mA} \mathrm{~cm}^{-2}$ with channel region (blue) and land region (red) marked

tural analysis of the cracks and blocks in the membrane. First we give a rough overview on how we convert the gray level images to black and white (BW) images with well-known methods of image analysis. To obtain quantitative numbers characterizing the structure of the cracks, the blocks need to be identified as objects from the BW image. The major part on the section describing the method addresses the 'radar' method which allows the determination of the width of the cracks between to neighbored blocks. Because those widths are measured at different locations the result is a collection of many numbers which can be analyzed statistically.

The cracks are analyzed for two regions of a membrane: one region under a gas channel and one region under the rib. The images were taken from radiographic projection images of a HT-PEFC operated at different electrical load levels. A systematic difference was found in the crack widths under the channels and under the ribs.

\section{Experimental}

\subsection{MEA preparation and cell performnance}

For the preparation of the gas diffusion electrodes (GDEs) a classical doctor blade technique was used. A homogeneous catalyst ink composed of carbonsupported catalyst (20\% HP Pt on Vulcan XC 72, BASF Fuel Cell, Inc.), PTFE (Dyneon, $40 \mathrm{wt} \%$ in the final catalyst layer) and different dispersants was coated onto a commercially available carbon non-woven gas diffusion layer (GDL) with a microporous layer on one side (H2315 C2, Freudenberg FCCT KG). The gas diffusion electrodes (GDEs) were produced with a platinum loading of $\sim 1 \mathrm{mg} \mathrm{cm}^{-2}$. After a drying step at room temperature the GDEs were doped with $20 \mathrm{mg} \mathrm{cm}^{-2}$ phosphoric acid per electrode. After the doping process the GDEs were joined with an undoped $30 \mu \mathrm{m}$ thick 
poly(2,5-benzimidazole) (ABPBI) membrane (FuMATech $\mathrm{GmbH}$ ) in a test cell without a preceding hotpressing step. The MEAs with an active area of $50 \mathrm{~cm}^{2}$ together with $300 \mu \mathrm{m}$ thick fluorinated polymer gaskets were compressed under the effect of a constant torsional moment of $5 \mathrm{Nm}$ between two graphitic single serpentine flow fields and massive metallic endplates equipped with two heating cartridges, each.

The cells were operated at $160{ }^{\circ} \mathrm{C}$ and ambient pressure. For all experiments dry gases were used and the mass flows of hydrogen and air for current densities equal to or greater than $j=140 \mathrm{~mA} \mathrm{~cm}^{-2}$ (typical cell voltage: $\sim 540 \mathrm{mV}$ ) have been adjusted to $\lambda_{\mathrm{An} / \mathrm{Ca}}=2 / 2$. At open circuit voltage $(\mathrm{OCV})$ the flow rates were adjusted to the same flow rates applied for $j=140 \mathrm{~mA} \mathrm{~cm}^{-2}$ at $\lambda_{\mathrm{An} / \mathrm{Ca}}=2 / 2$. A classical break-in procedure had been carried out prior to the synchrotron experiments detailed below. Therefore, the cell had been operated at $j=200 \mathrm{~mA} \mathrm{~cm}{ }^{-2}$ for $70 \mathrm{~h}$ in a test rig at the Forschungszentrum Jülich $\mathrm{GmbH}$, was then shut down and afterwards inserted into a mobile test rig at the BESSY II before performing the first synchrotron $\mathrm{X}$-ray measurements.

\subsection{Synchrotron $X$-ray radiography}

The radiographic measurements were performed at the synchrotron radiography station of the HelmholtzZentrum Berlin (BAMline at BESSY II). A monochromatic X-ray beam with an energy of $35 \mathrm{keV}$ was used to ensure a sufficiently high transmission through the cell. An optical setup with a $4008 \times 2672$ pixel CCD camera (PCO 4000 with a $\mathrm{CdWO}_{4}$ scintillator screen) was used to capture images with area sizes of about $8.5 \times 5.7 \mathrm{~mm}$ with a pixel size of $2.15 \mu \mathrm{m}$ resulting in an optical spatial resolution of approximately $5 \mu \mathrm{m}$ [18]. The exposure time per image was $8 \mathrm{~s}$, including a readout time of about $1.5 \mathrm{~s}$. In this paper we used images with $4008 \times 2122$ pixels showing an area of $8.5 \times 4.5 \mathrm{~mm}$.

A cell set-up with through-plane viewing direction that allows to distinguish between the land and the channels of the flow fields and to visualize the overlaid crack structures of the anodic and cathodic catalyst layers was chosen for all experiments.

For the statistical analysis we have taken radiography images from in-situ measurements on the described HTPEFC setup. An in-situ fuel cell was taken to analyze the structure of the MEA. Figure 2 shows a typical radiography of such a fuel cell operated at a mean current density of $600 \mathrm{~mA} \mathrm{~cm}^{-2}$.

The micro structure of the cracks originated by the manufacturing process of the electrodes can be seen very clearly in those images. In contrast to conventional images from light microcopy or SEM the radiography images show overlaid pictures of the cracks on both anode and cathode side.

\section{Mathematical methods}

Several methods were combined for analyzing the microstructure. First, images of the fuel cell were extracted from the three-dimensional synchrotron experiment at BESSY II. The result is a set of two-dimensional gray-level images. For structural analysis the gray level images first were converted into black/white (BW) images. Based on the BW images the structure can be analyzed. We concentrated our investigations on the analysis of the width of the cracks. For this purpose the radar method was applied which obtains the width of the cracks at many positions resulting in a distribution of cracks widths. At the end the distributions of cracks widths were analyzed with stochastic methods.

\subsection{Crack analysis}

The analysis of the cracks can be separated into three steps. First, the image has to be converted into a black/white image (binarization). This procedure is individually applied to every image and can be done using well-known methods of image preparation. The black pixels represent the blocks of the catalyst structure, the white ones are the cracks between them

In a second step the image is cleaned from small artifacts resulting from the BW conversion by applying erode and dilate operations. It is known that erode/dilate operations using the default $3 \times 3$ regions tend to have a larger impact in diagonal directions $\left(45^{\circ}\right)$ than in horizontal or vertical directions. As an example, round structures tend to distort to a diamond shape [19]. As a consequence the number of erode/dilate steps has to be kept low.

The third step is the structural analysis of the black/white images.

\subsection{Crack width with the radar method}

In Figure 2 it can be seen that the cracks do not have a constant width. Because of the irregularity of the morphological structure it is intended to get a distribution of crack widths taken at different locations in the structure.

First of all, the structure of blocks and cracks between them has to be identified from the image. The black/white image as shown in Figure 3 (left) contains the blocks and cracks. Structural information is defined by the individual blocks as objects and their shapes. The border of a block can be identified in a recursive way 


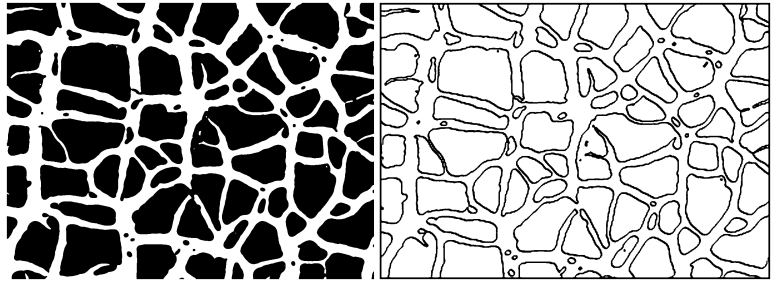

Figure 3: Black/white image of Figure 1with blocks (left) and borders of identified blocks after image processing (right)

starting from any pixel of the BW image which has a neighbor of different color. The borderlines are shown in the right part of Figure 3. Once a borderline is identified the corresponding block is collected in appropriate data structures. At the end, the blocks are given as a set of arrays containing the coordinates of the border pixels.

The set of borderlines from the above is the basis of the radar method to determine the widths of the cracks at many positions. At every $h$-th position $-h$ is a parameter of the radar method - the distance to the vis-a-vis block is determined and stored into an array.

The calculation of the distance to the vis-a-vis block first requires the calculation of a horizon which approximates the tangent of the border of the block at the current position $P$ of the borderline. The horizon is specified as red line in Figure 4. For determination of the horizon, first a circle of radius $r$ is drawn around the point $P$. This circle intersects the borderline at two points. The straight line through those points is the horizon from which the distance to the opposite block is calculated. With a suitable radius $r$ this line is an eligible measure of the orientation of the borderline of the block.

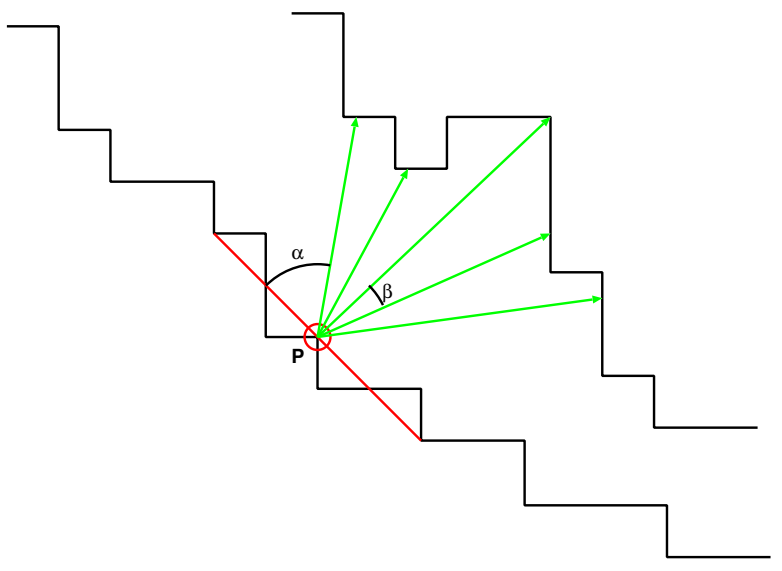

Figure 4: Principle of the radar algorithm

The distance between the starting point $P$ and the opposite block is determined as the minimal length of rays sent of from $P$ where they are intersecting the opposite borderline. Those rays are sent from $P$ in angles starting with $\alpha$ and rotating by $\beta$ each. Hence, the angles $\varphi$ between the horizon and the rays have all the form

$$
\varphi=\alpha+j \cdot \beta
$$

for the $j$-th ray. The situation is shown in Figure 4. The starting angle $\alpha$ has to be chosen carefully. When $\alpha$ is too small there is a risk that the rays might intersect the same block instead of the opposite one. On the other hand, a large $\alpha$ tends to a small range of angle covered by the rays. If for instance $\alpha$ is larger than $45^{\circ}$ then one might consider a situation of having a crossing. Let $P$ be on the edge of one block, let the horizon be calculated with an angle of $45^{\circ}$. With $\alpha$ larger then $45^{\circ}$ the shortest ray would hit the corner diagonal to the crossing. The shortest distance then would be the diagonal that does not apply the proper width of the crack.

The determination of the crack width is repeated for the next position at the border of the block with the step size $s$. The parameters of the radar method are summarized in Table 1 .

The choice of the offset angle $\beta=8.6^{\circ}$ leads to an error in the calculation of the distance less than $\cos (\beta / 2)<3 \%$. Irregular obstacles on the opposite border of a crack can be missed by the rays if their broadness $b$ is smaller than $1 / 13$ of the distance $d$ of the opposite border at the particular position. This limit can be estimated by $b / d<\sin (\beta / 2)$. E.g., at a position where the crack width is 27 pixels, a 2 pixel wide irregularity on the opposite border can be missed by two succeeding rays.

Figure 5 shows the application of the radar method to a crack shown in a section of a BW image. From every set of rays starting from one point the shortest one is collected into the set of numbers specifying the distribution of crack widths.

\section{Results}

A fuel cell with a flow field with channels of $1.5 \mathrm{~mm}$ width had been operated under five different conditions.

1. Operation without current - open circuit voltage (OCV) conditions

2. Operation with mean current density of $140 \mathrm{~mA} \mathrm{~cm}^{-2}$

3. Operation with mean current density of $350 \mathrm{~mA} \mathrm{~cm}^{-2}$

4. Operation with mean current density of $600 \mathrm{~mA} \mathrm{~cm}^{-2}$ 
Table 1: Parameters of the radar method

\begin{tabular}{ccl}
\hline Parameter & Value & Meaning \\
\hline$r$ & $3 \mathrm{px}$ & Radius for determination of the horizon \\
$\alpha$ & $30^{\circ}$ & Start angle for the radar method \\
$\beta$ & $8.6^{\circ}$ & Offset angle for rays - gives 15 rays from each position \\
$s$ & $10 \mathrm{px}$ & Step size for evaluation of the crack width \\
\hline
\end{tabular}

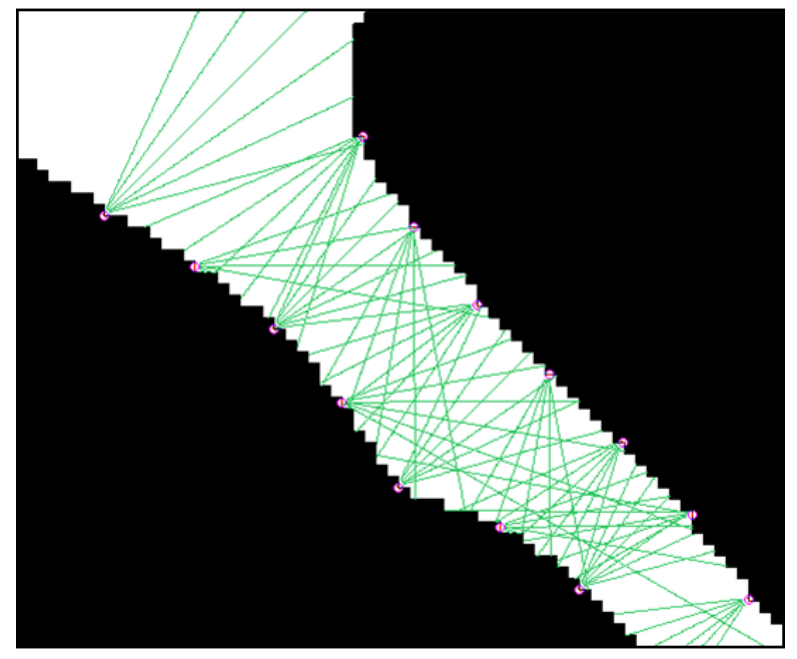

Figure 5: Radar method applied to a section of a BW image

5. Operation again under OCV conditions, labeled OCV2 in this article

Synchrotron images were created under these conditions, each of them were taken from the same location. The width of the cracks in the regions under the channels and under the ribs were analyzed separately. For this purpose, two regions of the synchrotron images were extracted as specified in Figure 2. The synchrotron images under the operating conditions mentioned above were separated in channel/land regions resulting in 10 images to be analyzed regarding crack widths.

The gray level images were converted into BW images using a threshold. A nested pair of erode/dilate steps of depth 2 (EEDD) followed of a nested pair of dilate/erode steps of depth 2 (DDEE) led to a satisfying result of the channel regions as shown in Figure 6 . The darker regions under the ribs were converted in a similar way. Fewer erode/dilate steps (EDDDEE) were needed to achieve the result shown in Figure 7.

While the previous steps are methods of classical image processing, the actual intent of our investigations is the extraction of morphologic information. With the radar method, distributions of the widths of the cracks

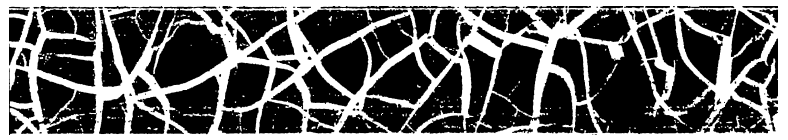

Figure 6: Channel region at $600 \mathrm{~mA} \mathrm{~cm}^{-2}$ after BW conversion

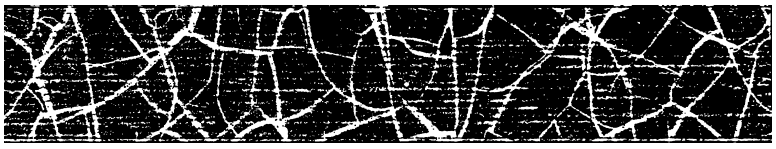

Figure 7: Land region at $600 \mathrm{~mA} \mathrm{~cm}^{-2}$ after BW conversion

were obtained from the 10 images - one for the channel region and one for the land region from every of the five operating conditions taken from the same region of the fuel cell.

Figure 8 shows detailed histograms of the crack widths from two images. The oversized heights of the leftmost bars in the histograms are due to the limited quality of the BW conversion, i.e. it is caused by the horizontal white stripes in Figures 6 and 7 . In despite of this source of irritation, it can already be seen from those diagrams that the distribution of the crack widths under the channels show a similar shape as the widths under the ribs of the bipolar plate. The summarized quantiles of the 10 images from all operating points are shown in Tables 2 and 3

Table 2: Characteristics of the crack widths $[\mu \mathrm{m}]$ of the channel region

\begin{tabular}{cccccccc}
\hline & Min & $25 \%$ & $50 \%$ & $75 \%$ & Max & mean & No. \\
\hline OCV & 12.05 & 14.91 & 22.65 & 49.91 & 165.18 & 34.96 & 4511 \\
140 & 12.05 & 14.91 & 22.24 & 49.56 & 163.34 & 32.12 & 4341 \\
350 & 12.05 & 14.91 & 19.64 & 44.87 & 160.72 & 32.94 & 4708 \\
600 & 12.05 & 14.91 & 23.43 & 51.31 & 160.72 & 36.29 & 4327 \\
OCV2 & 12.05 & 14.91 & 21.73 & 47.64 & 158.59 & 34.59 & 4337 \\
\hline
\end{tabular}

Table 2 shows the characteristics of the crack widths obtained from the region under the channel. The minimum and maximum values are shown as well as the quartiles of the data. The mean value is shown as well. All values are given in $\mu \mathrm{m}$. The right column of the table 

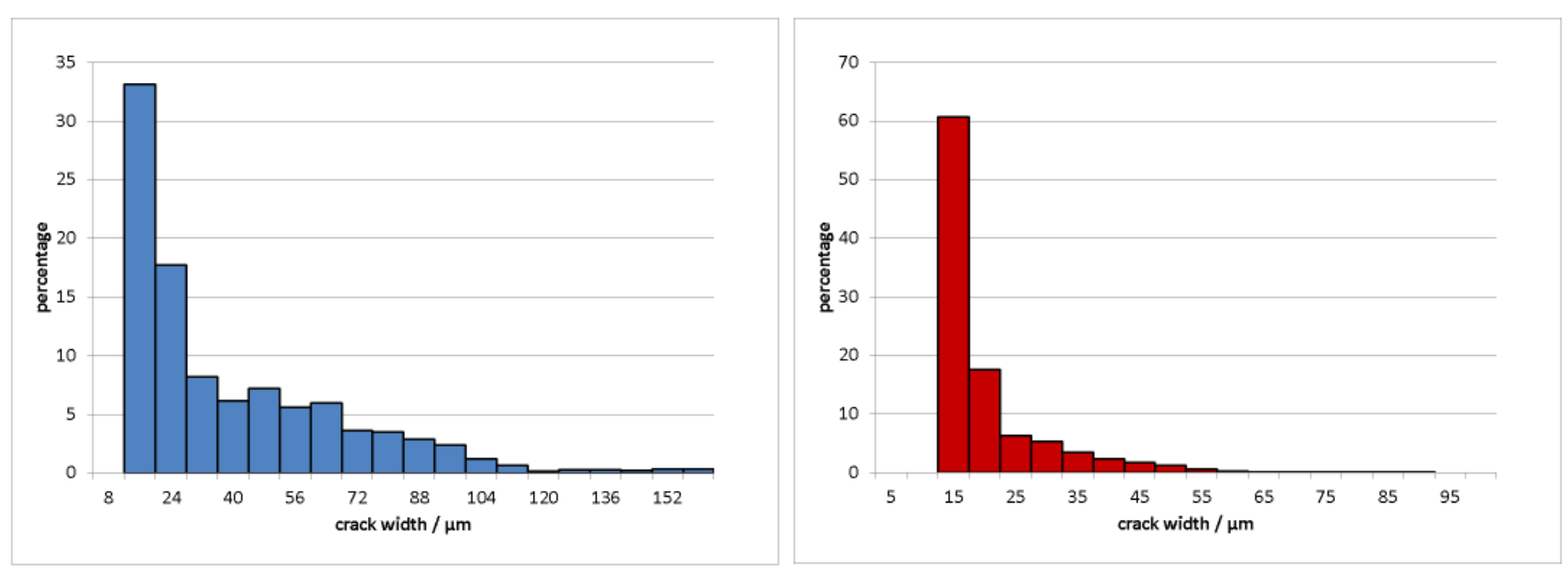

Figure 8: Crack width at $600 \mathrm{~mA} \mathrm{~cm}^{-2}$ in channel region (left) and land region (right)

Table 3: Characteristics of the crack widths $[\mu \mathrm{m}]$ of the land region

\begin{tabular}{cccccccc}
\hline & Min & $25 \%$ & $50 \%$ & $75 \%$ & Max & mean & No. \\
\hline OCV & 12.05 & 13.61 & 15.51 & 27.12 & 97.57 & 22.01 & 4294 \\
140 & 12.05 & 12.78 & 14.29 & 19.64 & 98.86 & 18.46 & 3484 \\
350 & 12.05 & 12.96 & 14.91 & 22.95 & 81.13 & 19.96 & 4936 \\
600 & 12.05 & 12.78 & 14.29 & 19.06 & 86.73 & 17.96 & 6621 \\
OCV2 & 12.05 & 12.78 & 14.29 & 19.06 & 86.29 & 17.92 & 6251
\end{tabular}

contains the number of evaluations of the crack widths in the data sets. The stochastic evaluation was applied to the data of the five operating points as they were observed by synchrotron radiography. Table 3 is the result of the stochastic evaluation on the land regions in the same way.

The Tables 2 and 3 show that the amount of data is large enough for a reliable stochastic analysis. From every image more than 3000 values were obtained. It has to be stated that the minimum values from tables obviously are dominated by the chosen method of image pre-processing to get the $\mathrm{BW}$ images. Also the first quartile - the $25 \%$ quantile - is dominated by the pre-processing. To confirm this effect, the following stochastic evaluation was performed on two sets of data. First, the complete data was taken for analysis taking into account the risk of a weakness in the results. Second, half the data - those above the second quartile - the $50 \%$ quantile - was chosen for evaluation.

The data from under the channels were compared with the data under the ribs from the same source. The Mann-Whitney U-test was chosen to compare the data. The assumption of this test is that both sets are representing data with the same shape of their distribution [20]. Because the cracks are originated from the man-

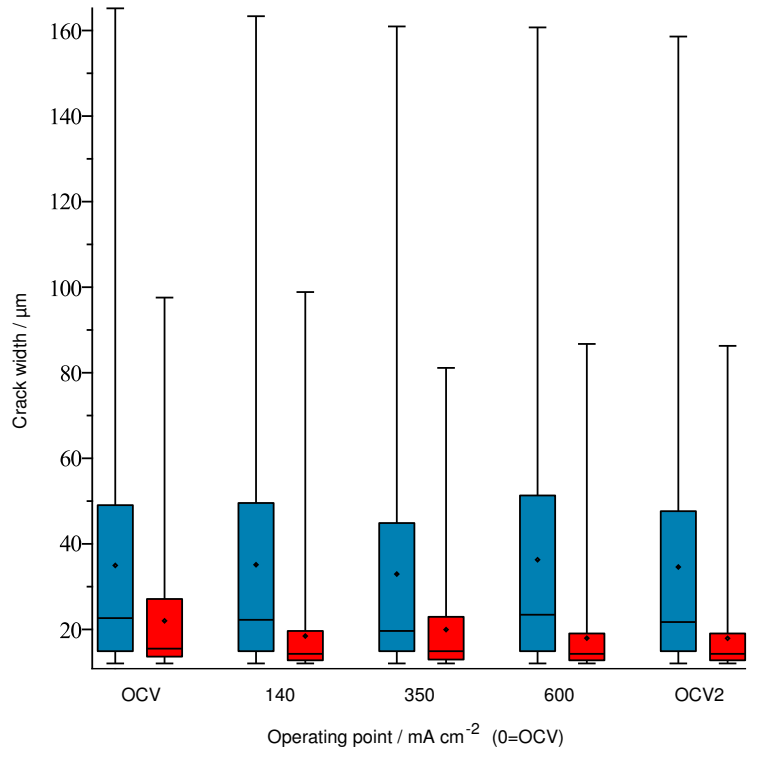

Figure 9: Boxplots of all images. Blue: region under the channel, red: region under the ribs. The bars specify the minimum and maximum values, the boxes show the quartiles of the distribution. The mean values are plotted as dots inside the boxes.

ufacturing process of the membrane, this assumption is most likely true. The histograms of the crack widths of the channel and land regions (Figure 8) give an additional approval of this fact which is substantiated by the large number of data items.

Table 4 shows the results of the Mann-Whitney Utest. The test can be applied to two data sets of arbitrary distribution - presumed that the distributions of the two data sets are of the same shape. The U-test was applied to two sets of data each under the hypothesis that 
Table 4: Mann-Whitney U test

\begin{tabular}{|c|c|c|c|c|c|}
\hline \multirow[t]{2}{*}{ Criterion } & \multicolumn{5}{|c|}{ Condition $/ \mathrm{mA} \mathrm{cm}^{-2}(0=\mathrm{OCV})$} \\
\hline & $\mathrm{OCV}$ & 140 & 350 & 600 & $\mathrm{OCV} 2$ \\
\hline$P\left(w_{c h}>w_{\text {rib }}\right)$ & 1 & 1 & 1 & 1 & 1 \\
\hline
\end{tabular}

the crack widths under the channel are larger than the crack widths under the rib. The result is the probability whether the values from the first data set which was taken from the channel region $\left(w_{c h}\right)$ are larger than the data from the second dataset which was obtained from the land region $\left(w_{r i b}\right)$.

In every case the U-test proves the impression which was already shown by the boxplots in Figure 9. The cracks under the channel are significantly wider than those under the ribs. The test method could show the statistical significance of a global relationship between the crack width distribution and their location. The crack widths under the rib are much smaller than under the channel. This can be derived from the blue bars for the quartiles of the channel region and the red bars for the region under the rib.

There are some additional effects shown in Figure 9 and Tables 2 and 3 . The minumum values and the $25 \%$ quantile are of the same value for nearly all data sets. This is due to inaccuracies in the BW conversion which are already indicated by the horizontal white stripes in Figures 6 and 7 and the large percentage values for the smallest crack widths in Figure 8 The crack widths at $350 \mathrm{~mA} \mathrm{~cm}^{-2}$ seem to be smaller than those at other operating conditions. That is indicated by the maximum value under the rib and the $75 \%$ quantile under the channel in Figure 9 Under the rib the cracks widths seem to be larger at OCV than at other operating conditions.

There are several reasons possible why an impact of the operating conditions was not confirmed by the Mann-Whitney U-test (except the trivial case of no relationship). On the one hand the crack widths might be dominated by limitations and artifacts of the BW conversion. On the other hand the U-test was applied to data of the whole area which does not allow analysis of regional effects.

The images at different load levels were visualized in the same way as published by Arlt et al. [21]. Similar qualitative effects could be observed in local areas of our synchrotron images. For this purpose the gray level images under electrical load were normalized to the OCV image, then restricted to the range from 0.94 to 1.08 . Figure 10 shows the normalized image of a local region under the channel, differences indicated as

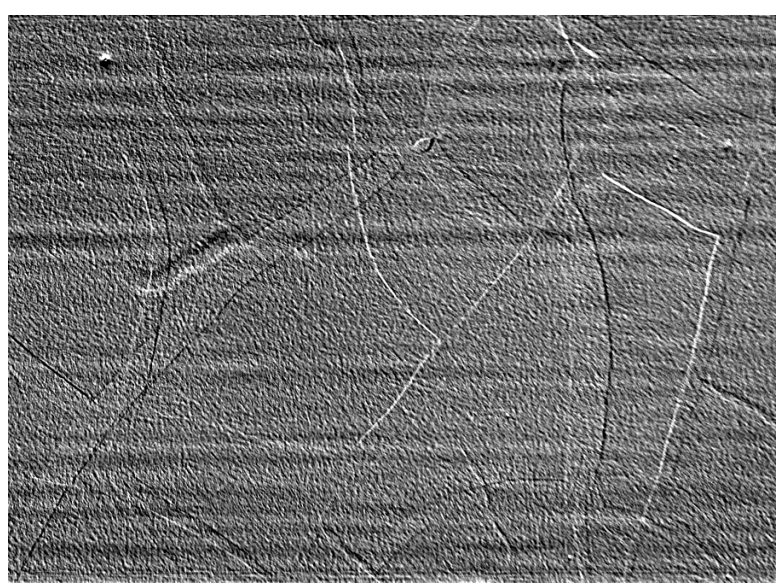

Figure 10: Local region under the channel $(1.9 \mathrm{~mm} \times 1.4 \mathrm{~mm})$ with morphological changes at $600 \mathrm{~mA} \mathrm{~cm}^{-2}$

white or black areas. White color in Figure 10 indicates areas where a crack is visible at $600 \mathrm{~mA} \mathrm{~cm}^{-2}$ but not under OCV condition. Black color indicates the opposite. The effects were observed in small regions. Furthermore, the white areas in Figure 10 mostly belong to one side of the membrane, the black areas belong to the other side. That can be concluded from tracking the cracks in the original image (Figure 2) starting at crossings of the cracks and comparing their locations with the white and black areas in the difference image. For this reason the impact of the operating conditions on the crack width can be analyzed only qualitatively. A more detailed quantitative analysis of the effects will require further development of image processing techniques and mathematical analysis methods.

\section{Conclusion}

Statistical methods were applied to calculate the crack width distributions in electrodes of a HT-PEFC during operation. The crack structure was detected via synchrotron X-ray radiography. The images taken from the in-situ measurements show structures from both anode and cathode side. It was shown that the cracks under the gas channels are significantly wider than under the ribs of the bipolar plate. Furthermore the crack width distribution strongly depends on the applied operating conditions.

The determination and analysis of morphological structures is a toolbox to analyze relationships between physical conditions and experimental observations. The established methods of X-ray radiography, image processing techniques, and the quantification of observations with statistical methods can complement each 
other in the investigation on structural changes of the dynamic behavior of fuel cell and other materials.

\section{Outlook}

In our future work we will enhance the image preprocessing techniques in order to reduce its influence on the calculated crack widths especially regarding the small values. Furthermore it is planned to develop an image analysis technique that allows distinguishing cracks on anode and cathode side and that enables us to compare the crack development on both sides separately.

\section{Acknowledgements}

The work was partly funded by The Danish Council for Strategic Research in the frame of the $4 \mathrm{M}$ project.

\section{References}

[1] Wannek C, Nehr S, Vahlenkamp M, Mergel J, Stolten D. Pseudo-half-cell measurements on symmetrical catalyst-coated membranes and their relevance for optimizing dmfc anodes. Journal of Applied Electrochemistry 2010;40:29-38.

[2] Bois C, Blayo A, Chaussy D, Vincent R, Mercier AG, Nayoze C. Catalyst layers for pemfc manufactured by flexography printing process: Performances and structure. Fuel Cells 2012;12(2):199-211.

[3] Swamy T, Kumbur EC, Mench MM. Characterization of interfacial structure in pefcs: Water storage and contact resistance model. Journal of The Electrochemical Society 2010;157(1):B77-85.

[4] Kundu S, Fowler MW, Simon LC, Grot S. Morphological features (defects) in fuel cell membrane electrode assemblies. Journal of Power Sources 2006;157:650-6.

[5] Markötter H, Haußmann J, Alink R, Tötzke C, Arlt T, Klages $\mathrm{M}$, et al. Influence of cracks in the microporous layer on the water distribution in a pem fuel cell investigated by synchrotron radiography. Electrochemistry Communications 2013;34:22-4.

[6] Manke I, Grünerbel M, Lehnert W, Kardjilov N, Haibel A Hilger A, et al. Investigation of water evolution and transport in fuel cells with high resolution synchrotron x-ray radiography. Applied Physics Letters 2007;90:174105.

[7] Sasabe T, Deevanhxay P, Tsushima S, Hirai S. Investigation on the effect of microstructure of proton exchange membrane fuel cell porous layers on liquid water behavior by soft $x$-ray radiography. Journal of Power Sources 2011;196:8197-206.

[8] Deevanhxay P, Sasabe T, Tsushima S, Hirai S. Observation of dynamic liquid water transport in the microporous layer and gas diffusion layer of an operating pem fuel cell by high-resolution soft X-ray radiography. Journal of Power Sources 2013;230:3843.

[9] Eller J, Roth J, Marone F, Stampanoni M, Wokaun A, Büchi FN. Implications of polymer electrolyte fuel cell exposure to synchrotron radiation on gas diffusion layer water distribution. Journal of Power Sources 2014;245:796-800.

[10] Novák V, Šimůnek J, van Genuchten MT. Infiltration into a swelling cracked clay soil. Journal of Hydromechanics 2002;50(1):3-19.
[11] Landry CJ, Karpyn ZT. Single-phase lattice boltzmann simulations of pore-scale flow in fractured permeable media. International Journal of Oil, Gas and Coal Technology 2012;5(2/3):182-206

[12] Hizir FE, Ural SO, Kumbur EC, Mench MM. Characterization of interfacial morphology in polymer electrolyte fuel cells: Micro-porous layer and catalyst layer surfaces. Journal of Power Sources 2010;195:3463-71.

[13] British standard 1134, method for the assessment of surface texture. 1972.

[14] Pfrang A, Veyret D, Janssen GJM, Tsotridis G. Imaging of membrane electrode assemblies of proton exchange membrane fuel cells by x-ray computed tomography. Journal of Power Sources 2011;196:5272-6.

[15] Mulone V, Karan K. Analysis of capillary flow driven model for water transport in pefc cathode catalyst layer: Consideration of mixed wettability and pore size distribution. International Journal of Hydrogen Energy 2013;38:558-69.

[16] Ko J, Ju H. Effects of cathode catalyst layer design parameters on cold start behavior of polymer electrolyte fuel cells (pefcs). International Journal of Hydrogen Energy 2013;38:682-91.

[17] Arlt T, Manke I, Wippermann K, Riesemeier H, Mergel J, Banhart J. Investigation of the local catalyst distribution in an aged direct methanol fuel cell mea by means of differential synchrotron x-ray absorption edge imaging with high energy resolution. Journal of Power Sources 2013;221:210-6.

[18] Maier W, Arlt T, Wannek C, Manke I, Riesemeier H, Krüger P, et al. In-situ synchrotron $\mathrm{X}$-ray radiography on high temperature polymer electrolyte fuel cells. Electrochemistry Communications 2010;12:1436-8.

[19] Russ JC. The image processing handbook. 6 ed.; CRC press; 2011.

[20] Sachs L. Angewandte Statistik. 9 ed.; Springer; 1999.

[21] Arlt T, Maier W, Tötzke C, Wannek C, Markötter H, Wieder F, et al. Synchrotron x-ray radioscopic in situ study of hightemperature polymer electrolyte fuel cells - effect of operation conditions on structure of membrane. Journal of Power Sources 2014;246:290-8. 